\title{
Poly-alendronate Dextran-Guanidine Conjugate
}

National Cancer Institute

\section{Source}

National Cancer Institute. Poly-alendronate Dextran-Guanidine Conjugate. NCI

Thesaurus. Code C101894.

A polybisphosphonate dextran-guanidine conjug ate with potential anti-resorptive and antineoplastic activities. Alendronic acid and aminoguanidine were conjug ated sequentially to oxidized dextran resulting in an average of 8 alendronate and 50 guanidine groups coupled to the dextran backbone. Upon administration, the poly-alendronate dextran-guanidine conjug ate inhibits the mevalonate pathway by inhibiting farnesyl diphosphate synthase (FDPS) which leads to a reduction in protein prenylation and to the loss of downstream metabolites essential for osteoclast function. This eventually leads to the induction of apoptosis in osteoclasts. Also, by preventing osteoclast-mediated bone resorption, this agent decreases bone turnover and stabilizes the bone matrix. The guanidine moiety increases the nitrogen content and possibly the activity of the bisphosphonate and its ability to inhibit FDPS. In addition, the guanidine moiety facilitates cell internalization and may contribute to this agent's cytotoxicity. 